\title{
Application of Cloud Manufacturing based on the principle of Process- Aware Information System
}

\author{
Ma Ping \\ School of Computer Science and Engineering \\ Beifang University of Nationalities \\ Yinchuan, Ningxia, China \\ e-mail:mapin_88@163.com \\ Bao Wen-xing \\ School of Computer Science and Engineering \\ Beifang University of Nationalities \\ Yinchuan, Ningxia, China \\ e-mail: bwx71@163.com
}

\author{
Han Qiang \\ School of Computer Science and Engineering \\ Beifang University of Nationalities \\ Yinchuan, Ningxia, China \\ e-mail: hanqiang9@163.com \\ zhang xiao-wu \\ School of Computer Science and Engineering \\ Beifang University of Nationalities \\ Yinchuan, Ningxia, China \\ e-mail: zhangxia_4043@qq.com
}

\begin{abstract}
Cloud computing refers to the computer platform technology, via the network with the way of on-demand and extendibility to get the required service. The core value of cloud computing is its on-demand scalability and the application value of enterprise private cloud is that it can cover the entire production process. Meanwhile, the application of cloud computing in manufacturing area simply referred to as cloud manufacturing and it is a new model and new way for manufacturing information technology development. As an important application field of cloud computing, this paper introduces composition and connotation of cloud manufacturing application system based on cloud manufacturing hierarchy model. Our work also has presented the cloud manufacturing system architecture model and an application solution based process -aware information system.
\end{abstract}

Keywords-Cloud computing; Cloud manufacturing; Internet of things; Process-Aware

\section{INTRODUCTION}

With the comprehensive application of the Internet, the coverage which manufacturing company focusing on product's information activities and process control has expanded rapidly, and the trend that parallel operation for companies' multiple information system and integrated centralized control has emerged. Simultaneously, the development of IOT (Internet of Things) technology has promoted the integration of process-aware technology and wireless sensor networks, and opened a new stage that manufacturing company can have a bottom-up physical processes-aware and large-scale data processing. These two advances inspire the enterprise information system more comprehensive and deeper interoperability and promote the manufacturing enterprises' private cloud computing --cloud manufacturing technology's research, development and application [1].

In recent years, the implementation experience from ministry of science's manufacturing information technology engineering topic shows, manufacturing process' real-time detection and control capabilities determine the allocation of manufacturing resources and execution capability in enterprise' manufacturing. Because of this discovery, Chinese scholars put forward the innovative idea, using cloud computing technology and put network and physical process together to achieve virtualization and service-oriented of all kinds of manufacturing resources and manufacturing capabilities as well as implementing a unified and centralized intellective management and business, which is called cloud manufacturing [2]. It enriches and expands the content of cloud computing resource sharing and service models and it also is an important priority development area of cloud computing industry.

Discrete manufacturing company should treat introducing IOT technology in the production management as the starting point, build enterprise manufacturing IOT systems, and develop production process sensing capability. On the basis of the enterprises' existing integration of multiple systems in parallel, doing cloud services structured treatment in computer center's hardware, database, user interfaces and application environment, achieving enterprise computer hardware cluster application and information system software resources virtualization, Supporting enterprises to gain greater customization and organizational control, to accelerate transformation and upgrading of company.

\section{CLOUD MANUFACTURING APPLICTION MODELS}

\section{A. The application models of cloud computing have been expanded by cloud Manufacturing technologies.}

Cloud computing is the higher level of "centralized" computing model, which is based on the parallel and distributed computing technology [3]. Li Bohu, academician of CAE (Chinese Academy of Engineering), propose that cloud manufacturing is a web-based, service- 
oriented intelligent manufacturing model [4]. Cloud manufacturing will provide a series of cloud service, which is more complete and in-depth and wisdom, based on the theoretical framework in cloud computing of computer science and technology, involving, Argumentation as a Service, Design as a Service, Fabrication as a Service, Experiment as a Service, Simulation as a Service, Management as a Service, Operation as a Service, Integration as a Service and so on [5].

Construction and application of cloud manufacturing made new demands on cloud computing technology. (1) Demand dynamic architecture--according to user needs, providing manufacturing services anywhere and at anytime. (2) Interoperability for collaborative-- businessoriented multiple user access, large and complex manufacturing tasks coordination, supporting interoperability between manufacturing capabilities and manufacturing resource.(3) Heterogeneous integration for physical processes underlying information-- supporting for distributed heterogeneous manufacturing resources and capabilities integration.(4) Powerful, fast, unlimited capacity--composing various services in response to demand quickly and flexibly [6].

Cloud manufacturing application model, designed to enable enterprises' private cloud users to work without interact directly with each resource and sensor, so users could get customizable virtual manufacturing environment and manufacturing capabilities from virtualized manufacturing resource pools through their terminal. The main cloud manufacturing application model has, (1) supports single subject to complete a stage of the manufacturing tasks (such as innovation design). (2) Support for completing a stage of manufacture task with multi-agent collaboration (such as design for quality). (3) Supports for completing cross-phase stage manufacturing tasks with multi-agent coordination (such as serialized and combinatory design, etc.) [5].

\section{B. Supporting Technologies in the Cloud Manufacturing Models}

To achieve the above application pattern of cloud manufacturing, foundation should be manufacturing resource and capacity being instrumented. Namely using advanced IOT (Internet of Things) technology as the cloud manufacturing infrastructure and achieve the integration of hardware and software resources, capabilities, information physical processes [7]. By information perception and wireless sensor networks access of whole system, whole life cycle and a full range, we can achieve instrumentation of manufacturing resources and capabilities [8]. Introducing cloud computing virtualization technology, to make each of the physical manufacturing resources and capabilities performance for the cluster virtual device of interacting the physical environment 、 hardware and software. Using the server powerful processing capability generates multiple virtual servers to support a higher level, the larger granularity of virtual manufacturing unit. By desktop virtualization technology, users in different place and with different devices can receive service which is virtualization manufacturing resources and capabilities live migration and dynamic scheduling. So as to effectively support the full life cycle of manufacturing companies' person /organization, management and technology integration and optimization.

Since the lack of continuous manufacturing environment

like process industries in discrete manufacturing, so relying solely on improving the level of automation equipment or blindly enhance enterprise management information tools can not achieve agility and flexibility in production process. Nor lasting improvement companies' quickly respond to market changes and customer needs. However, a medium-sized discrete manufacturing enterprise, the number of parts including products and finished products that it produced each year could up to as many as one million, companies manufacturing execution system used for management and manufacturing for these products should make one hundred million data tests from the production process. Such data has to rely on advanced and powerful computer systems processing. Enterprise IOT necessarily requires cloud computing in cloud manufacturing system to centralized process distributed data.

Manufacturing enterprises' IOT generally have a lot of sensors and a large number of collected production process data, these data need to achieve distributed real-time storage and aggregation to centralized computing facilities by wireless sensor networks, broadband internet. Therefore, the use of cloud computing to host these tasks will have a significant cost advantage [9].Cloud represents the network and the networking, and computing represents a computer-related resources, applications and services. IOT can help companies achieve low-cost data acquisition, transfer and use from production site. No cloud computing, massive data in production process will be difficult to digest, convert, statistics, forecasting and control.

Moreover, manufacturing enterprises' production equipment disperse during operation, technology and processes were irregular in spatial distribution, so enterprise must integrate concentrate - distributed IT resources, formed a certain scale, low-cost, dynamically scalable and parallel, fault-tolerant computing data centers, only in this way, can support massive data analysis and calculations, including the intelligence aggregation of many multi-source perception information, excavation and analysis etc.

Therefore, take cloud computing as the manufacturing IOT's supporting platform is necessary. It is expected that in the manufacturing sector, IOT will become the largest user of cloud computing, and cloud-oriented manufacturing is the competitiveness for manufacturing enterprise which can not be copied.

\section{Cloud MANUfACTURING BASED ON PROCESS- AWARE INFORMATION SYSTEM}

The challenge of today's manufacturing information is no longer how to encode a single module, but how to 
transform from 'data-oriented coding - assembly' system development model to the 'process-oriented perception control' integrated service model [10]. Therefore, the development of cloud manufacturing systems need to be synchronized with the development of enterprise manufacturing systems and manufacturing environment, and support overall perception for products, resources, information, organizational elements in the manufacturing process to the manufacturing enterprises which is as the core of the supply chain. Naturally, achieve enterprise multi-source information integration via cloud manufacturing systems, so enterprise manufacture system could adapt to internal and external environment which is complexity and variability.

\section{A. Conceptual design of the system architecture}

Cloud manufacturing system, the key technology is how to deal with the underlying perception of events, to form event-driven process-aware information systems function. Including, (1) the architecture based on event- driven for cloud manufacturing system.(2) Distributed computing and process-aware information flow integration and interaction based on publish - subscribe model. (3) Timing choice and control of production logistics detection data and wireless network asynchronous communication pattern. (4) Coupled drive relations between job shop scheduling decisions and process-aware event, etc.

In this paper, by the use of the basic principle of PAIS (Process Aware Information Systems), (System customers interact with information systems through switching products, and achieve production and assemble for products, which is participation by participants, information, technology)suggests the hierarchical structure correspond exactly to the theory of academician Li Bohu's cloud manufacturing systems and shows the small-medium enterprise cloud manufacturing system architecture model constituted by six entities[4][11]. As shown in Table 1 and Figure 1.

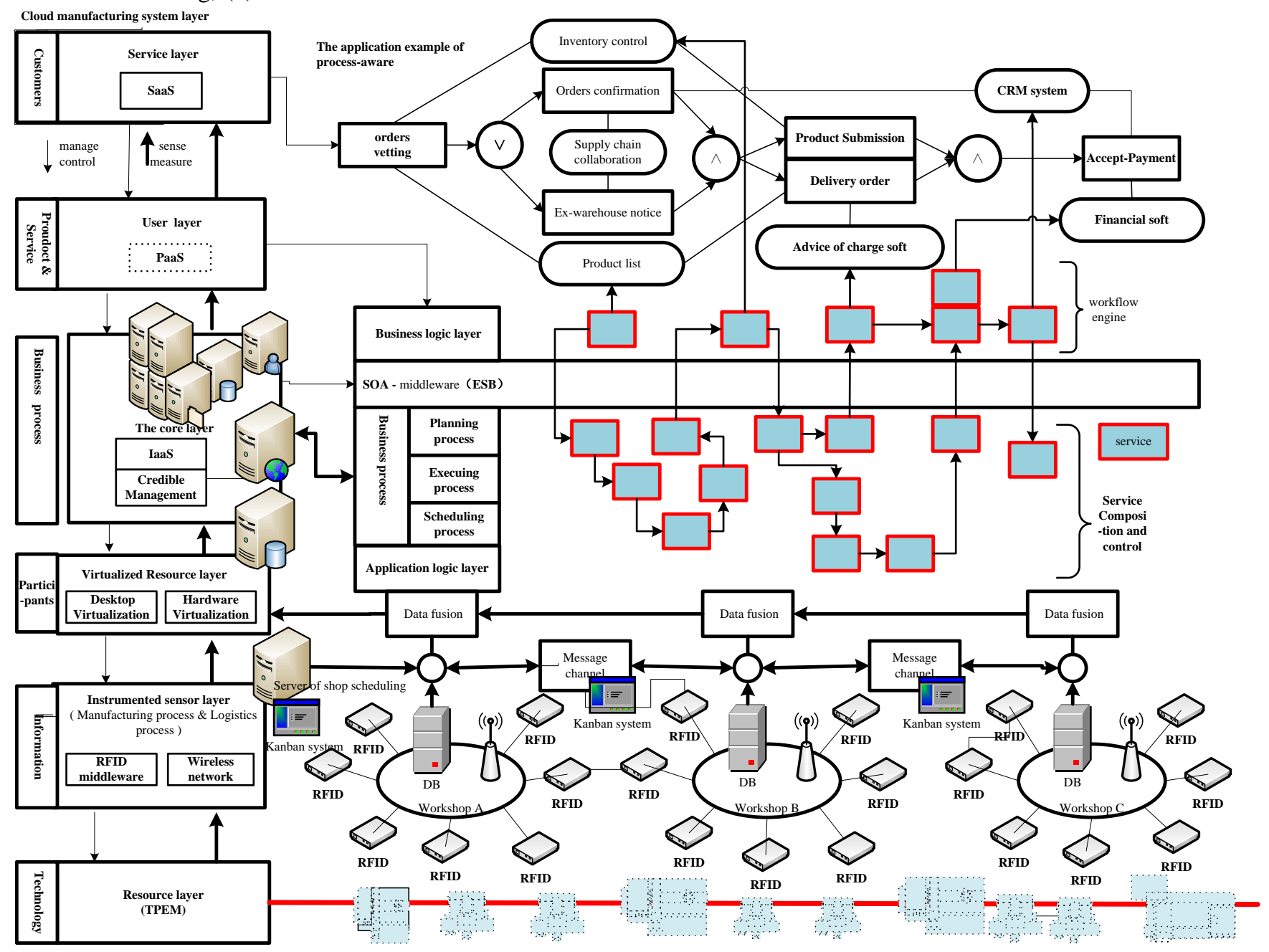

Figure 1. The general structure cloud manufacturing based on the principles of process-aware information system. 
TABLE I. CORRESPONDING RELATIONSHIP

\begin{tabular}{|c|c|c|}
\hline layer & $\begin{array}{l}\text { Cloud manufacturing } \\
\text { system layer }\end{array}$ & $\begin{array}{c}\text { Process-Aware } \\
\text { Information System layer }\end{array}$ \\
\hline 1 & Service layer & Customers \\
\hline 2 & User layer & Products and Services \\
\hline 3 & The core layer & Business Processes \\
\hline 4 & $\begin{array}{l}\text { Virtualized Resource Layer } \\
\text { (IT resource and } \\
\text { Manufacturing capacity) }\end{array}$ & Participants \\
\hline 5 & $\begin{array}{l}\text { Instrumented Sensor Layer } \\
\text { ( RFID and Wireless sensor } \\
\text { networks) }\end{array}$ & Information \\
\hline 6 & Resource Layer & Technology \\
\hline
\end{tabular}

Table 1 shows, cloud manufacturing system level can put the process-aware information system's core concepts into products and services, so they could achieve synchronization and balanced expansion for IT infrastructure, thereby they could be able to cope with challenges for manufacturing enterprise with the new architecture.

Corresponding to the cloud manufacturing system level is PAIS level, it showing, customers interact with information systems through switching products or services, product assembly via business process state which is participation by information and technology perceived from participants, and then tells to users, and users could do management and control. Participant includes virtualized hardware and software. Technology represents the ways of working which is needed by manufacturing system for the completion of all required business processes [11].

Figure 1 is an application example which cloud manufacturing system based on the principles of PAIS. And it is specifically describe in Table 1 . Figure 1 shows, Cloud manufacturing system can realize interconnection and combination for manufacturing enterprise multi-level application software modules. Enterprises take the form of a virtual organization based on cloud computing technology to build a new information processing capabilities in the spacetime, logical-physical, software-hardware, resource-ability of these four key areas and get a bottom-up new integration from distributed, parallel processes and organization.

\section{B. Manufacturing IOT System Virtualization Solution}

The task of manufacturing processes underlying architecture is to detect the production process status information which production equipment status and production logistics status. Its features are based on RFID middleware and wireless network operating system implementation, (1) Distributed parallel information collection based wireless network. (2)Achieve integration application of physical sensory information and business logic information via middleware, including function of RFID sensory information received and stored in the database, achieving bottom-up resource sharing between multilayer technical interfaces systems and hierarchies.(3)
Reconstruct the enterprise underlying 'material - material, matter - people, objects - bit' new causal relationship based on information fusion technology and artificial intelligence techniques in different time sequences. Therefore, wireless sensor network system which commitments workshop bottom manufacturing IOT capabilities is a network related to application tasks and its design, deployment and management should be subject to specific production process measurement and control demand.

However, because of the large number of nodes and limited resources in wireless sensor networks and the harsh environment in monitoring area, so it is very essential to take the wireless networks management into the cloud manufacturing system and to develop effective operation IOT wireless networks management strategy of manufacturing IOT system for planning, deployment and maintenance workshop wireless networks.

In recent years, a number of advanced enterprises have established a relatively complete virtual manufacturing systems, the computer can simulate the real material flow, information flow and value stream manufacturing system. With establishment and application of cloud manufacturing systems, enterprises' management began to focus on the manufacturing process state gathering and they hope to get the complete picture of underlying information physical processes timely using networking technology and interfere in physical process via application software's information processing flow. To this end, in order to realize the underlying information physical system virtualization management and control on the manufacturing process, it is required to investigate how to take the cloud computing virtualization technology into a platform for enterprise systems underlying technology.

From the show of bottom layout in Figure 1, the directly support of running RFID IOT manufacturing system and wireless sensor networks system is distributed production database which accepts workshop scheduling server management and service. Accordingly, we can deploy multiple virtual machines on the workshop scheduling server and each virtual machine corresponding to a workshop. Networks virtualization can realize using vNetwork elements in vMware vSphere and using the vCloud Director pooling mechanism [12]. Realizing distributed management for wireless networks in various workshops via VLAN label. RFID reader devices virtualization could regard its location (ID) as the label through the virtual storage to achieve its virtualization.

By the virtualization to RFID middleware and wireless network operating system, it can directly visualize the realtime status information flow in production process detection for entity such as progress and equipment. And workshop job scheduling software could play a greater role. Enterprise underlying perception virtualization is conductive to management to use distributed data resource and reduce costs of massive data management and maintenance. Then enterprise cloud manufacture data center is responsible for analysis historical data and high-level applications. 


\section{CONCLUSION}

The use of PAIS in cloud manufacturing system could carry out construction and optimization for manufacturing resources and manufacturing capacity with the company's own manufacturing information physical processes closely integrated. And then put various manufacturing resources and manufacturing capabilities virtualization, serviceoriented with cloud computing, continuously improve the manufacturing system information physical processes in the application. Also promote the transition from a single component production model to combination production model in enterprise products manufacture.

With the development of cloud manufacturing, enterprises' private clouds will develop to hybrid clouds. And make the same kind of small and medium-sized enterprises access to manufacturing resources and service at any time on-demand through the terminal and the network. IT is beneficial to promote enterprise business model innovation. At the same time, in the process of developing a private cloud, enterprises should develop standardized, customizable outgoing service supply capacity, actively drive and set up cloud manufacturing construction and implementation of industry standards, trying to provide private cloud manufacturing services to enterprise in the same industry, stimulating supply chain related enterprise collaborative manufacturing with low cost software services. Ultimately, enhance the value of cloud computing platform significantly and promote industry technological advance.

\section{ACKNOWLEDGMENT}

Our work is a research project of Beifang University of Nationalities (No. 2013XYZ030). This work was supported by Beifang University of Nationalities grant. I would like to thank all team members for their assistance in developing this article.

\section{REFERENCES}

[1] Schaefer, D.J.L. Thames, R. Wellman, D. Wu, S. Yim, D. Rosen (2012). "Distributed Collaborative Design and Manufacture in the Cloud--Motivation, Infrastructure, and Education". Proceedings of the ASEE 2012 Annual Conference and Exposition, San Antonio, Texas, June 10-13, 2012, Paper No. AC2012-3017Electronic Publication: Digital Object Identifiers (DOIs).

[2] “Computer Integrated Manufacturing Systems Conference Report", PPT document. Huazhong University of Science, Nov. 2012.

[3] Huang Kai, Fox, Dongara with Wu Yong Wei, "Cloud Computing and Distributed Systems, From Parallel Processing to the Internet of Things",M. Machinery Industry Press, Jan. 2013.

[4] Li Bohu, ZHang Lin, Wang Shilong, "Cloud manufacturing: a new service-oriented manufacturing model", J.Computer Integrated Manufacturing Systems, 2010, 16(1):1-7.

[5] Li Bohu, ZHang Lin, Ren Lei, "Further discussion on cloud manufacturing",J.Computer Integrated Manufacturing Systems, 2011,17(3):449-457.

[6] D.Wu, L.Thames, D. Rosen, D. Schaefer, (2012). "Towards a CloudBased Design and Manufacturing Paradigm, Looking Backward, Looking Forward", Proceedings of the ASME 2012 International Design Engineering Technical Conference \& Computers and Information in Engineering Conference, Paper\#DETC2012-70780, Chicago, U.S.

[7] L. Atzori, A. Iera, G. Morabito, "The Internet of Things", A survey, Comput Netw. 54 (2010) 2787-2805.

[8] V. Kulkarni, A. Förster, G.K. Venayagamoorthy, "Computational Intelligence in Wireless Sensor Networks", A Survey, IEEE Communications Surveys \& Tutorials. 13 (2011) 68-96.

[9] Liu Peng, "Cloud Computing (Second Edition)",M.Electronic Industry Press , May 2011.

[10] Li Hao,Ji Yang-jian,Qi Guo-ning,Gu Xin-jian,Tang Ren-zhong, "Connotation,theory and key technologies system on the fusion of manufacturing and services", J.Computer Integrated Manufacturing Systems, 2010,16(11):2521-2528.

[11] Marlon Dumas, Wil M. van der Aalst, Arthur H. ter Hofstede, "Process-Aware Information System", M. Tsinghua University press, Feb.2009.

[12] R.Buyya, C.S.Yeo, S. Venugopal, J.Broberg, I.Brandic, "Cloud computing and emerging IT platforms, vision, hype, and reality for delivering computing as the 5th utility", Future Gener Comp Sy. 25(2009) 599-616. 